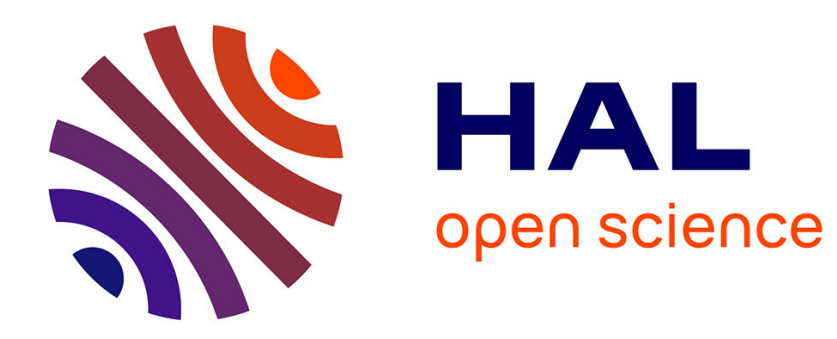

\title{
Propagation by pairing excitations in open shells
} F.J.W. Hahne, H.B. Geyer

\section{To cite this version:}

F.J.W. Hahne, H.B. Geyer. Propagation by pairing excitations in open shells. Journal de Physique Lettres, 1978, 39 (24), pp.479-481. 10.1051/jphyslet:019780039024047900 . jpa-00231550

\section{HAL Id: jpa-00231550 https://hal.science/jpa-00231550}

Submitted on 1 Jan 1978

HAL is a multi-disciplinary open access archive for the deposit and dissemination of scientific research documents, whether they are published or not. The documents may come from teaching and research institutions in France or abroad, or from public or private research centers.
L'archive ouverte pluridisciplinaire HAL, est destinée au dépôt et à la diffusion de documents scientifiques de niveau recherche, publiés ou non, émanant des établissements d'enseignement et de recherche français ou étrangers, des laboratoires publics ou privés. 


\title{
PROPAGATION BY PAIRING EXCITATIONS IN OPEN SHELLS
}

\author{
F. J. W. HAHNE and H. B. GEYER \\ Physics Division, Atomic Energy Board, Pelindaba 0001, Transvaal, South Africa
}

(Reçu le 29 août 1978, accepté le 24 octobre 1978)

\begin{abstract}
Résumé. - La méthode de la fonction de propagation des excitations fondamentales est appliquée au cas de trois particules en dehors d'une couche saturée. Il est démontré que la fonction de propagation ne contient pas de contributions parasites. Le fait que la base est surabondante a l'avantage de permettre l'extraction de coefficients de parentage fractionné.
\end{abstract}

Abstract. - The propagator method for elementary excitations is applied to three particles outside a closed shell. It is shown that the propagator contains no spurious contributions and that the overcompleteness of the basis has the advantage that coefficients of fractional parentage can be extracted.

A method of describing nuclear states by means of phonon-particle and pairon-hole propagators was recently proposed [1.2] and shown to be much simpler than nuclear field theory [3]. The latter theory has now been applied to three particles outside a closed shell where the angular momentum algebra has been done explicitly and some simple examples have been discussed to demonstrate how spurious states are eliminated [4]. In the present paper we show that the solution of the above-mentioned problem is not only easier accomplished by our propagator approach [1. 2]. but is also physically more transparent than nuclear field theory $[3,4]$.

The model hamiltonian is

$$
\mathscr{H}=\sum_{p} \varepsilon_{p} a_{p}^{+} a_{p}+\sum_{\left(p_{1} p_{2}\right)\left(p_{3} p_{4}\right)} V_{p_{1} p_{2} p_{3} p_{4}} a_{p_{1}}^{+} a_{p_{2}}^{+} a_{p_{4}} a_{p_{3}}
$$

where $V_{p_{1} p_{2} p_{3} p_{4}}$ is an anti-symmetric particle-particle interaction and the pairon creation operator is introduced as

$$
P_{\pi}^{+}=\sum_{\left(p_{1} p_{2}\right)} Z_{p_{1} p_{2}}^{\pi} a_{p_{1}}^{+} a_{p_{2}}^{+}
$$

The brackets indicate that the sum is restricted to ordered pairs. The pairon energy is $\omega_{\pi}$.

Applying the equations of motion to the particlepairon propagator

$$
G_{p_{1} p_{2}}^{\pi_{1} \pi_{2}}(t)=\left\langle 0\left|P_{\pi_{1}}(t) a_{p_{1}}(t) a_{p_{2}}^{+}(0) P_{\pi_{2}}^{+}(0)\right| 0\right\rangle \theta(t)
$$

leads to the Dyson-like equation [1. 2]

$$
\begin{aligned}
G_{p_{1} p_{2}}^{\pi_{1} \pi_{2}}(\omega)=G_{0 p_{1}}^{\pi_{1}}(\omega) & {\left[\delta_{p_{1} p_{2}} \delta_{\pi_{1} \pi_{2}}+X_{p_{1} p_{2}}^{\pi_{1} \pi_{2}}\right]+} \\
& +\sum_{p_{3} \pi_{3}} G_{0 p_{1}}^{\pi_{1}}(\omega) M_{p_{1} p_{3}}^{\pi_{1} \pi_{3}} G_{p_{3} p_{2}}^{\pi_{3} \pi_{2}}(\omega)
\end{aligned}
$$

where

$$
\begin{aligned}
X_{p_{1} p_{2}}^{\pi_{1} \pi_{2}} & =-\sum_{p_{3}} Z_{p_{2} p_{3}}^{\pi_{1}} Z_{p_{1} p_{3}}^{\pi_{2}} . \\
M_{p_{1} p_{2}}^{\pi_{1} \pi_{2}} & =\sum_{p_{6}\left(p_{7} p_{8}\right)} Z_{p_{6} p_{2}}^{\pi_{1}} V_{p_{1} p_{6} p_{7} p_{8}} Z_{p_{7} p_{8}}^{\pi_{2}} . \\
G_{0 p_{1}}^{\pi_{1}}(\omega) & =1 /\left(\omega-\varepsilon_{p_{1}}-\omega_{\pi_{1}}\right) .
\end{aligned}
$$

The expressions (4) and (5) are illustrated graphically in figure 1. These expressions can easily be interpreted in the $m$-scheme. However. if we couple a pairon with $\lambda \mu$ and a particle with $j m$ to total angular momentum $I M$, we find

and

$$
\left\langle j_{1} i I M|X| j_{2} i^{\prime} I M\right\rangle=-\sum_{j_{3}} \hat{i}^{\prime} \hat{\imath}^{\prime}\left\{\begin{array}{ccc}
j_{2} & j_{3} & \lambda \\
j_{1} & I & \lambda^{\prime}
\end{array}\right\}(-1)^{\hat{\lambda}-\hat{i}^{\prime}+2 j_{3}+j_{2}-j_{1}} Z_{j_{2} j_{3}}^{\dot{\lambda}} Z_{j_{j_{1} j_{3}}^{\prime}}^{\hat{\prime}^{\prime}}
$$

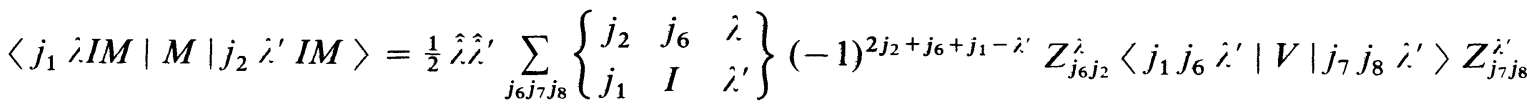



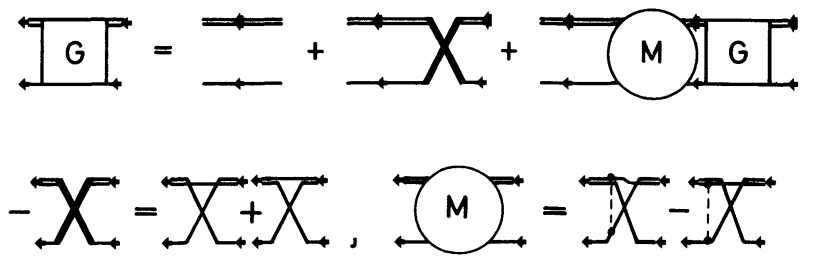

Fig. 1. - The pairon-particle propagator. Long single and double lines represent the propagation of particles and pairons, respectively; short lines represent labels only. The interaction $V$ is indicated by two dots connected with a broken line: the exchange part is not shown. The top line in the pairon refers to the first index.

with $\hat{\lambda}=(2 \lambda+1)^{1 / 2}$. The normalization of the pairon wavefunction $Z_{j_{1} j_{2}}^{\lambda}$ is

$$
\sum_{j_{1} j_{2}}\left(Z_{j_{1} j_{2}}^{\lambda}\right)^{2}=2
$$

because the sum is not ordered.

Using equations (6) and (7) the propagator $G$ can be calculated in the coupled representation $I M$. We apply it here to the single-shell case discussed in reference [4]. From equation (8) we then have

$$
Z_{j j}^{\lambda}=\sqrt{2} .
$$

The pairons assume angular momentum values $\lambda=0, \ldots .2 j-1$.

Taking $I=3 j-4$. only pairons with $\lambda_{1}=2 j-1$ and $\lambda_{2}=2 j-3$ contribute and for these values the diagonal parts of the $6 j$-symbol (when viewed as a matrix with indices $\lambda_{1}, \lambda_{2}$ ) appearing in $X$ are

$$
-1 /(8 j-2) \text { and }-1 /(8 j-10) \text {. }
$$

respectively. The non-diagonal parts vanish. Thus $1+X$ vanishes identically implying that the function $G(\omega)$ vanishes. This shows that no state with $I=3 j-4$ exists. Turning to states with $I=3 j-3$. we again have to consider only two $\lambda$-values.

$$
\lambda_{1}=2 j-1 \text { and } \lambda_{2}=2 j-3 .
$$

For these, the $6 j$-symbols give

$$
\begin{aligned}
& \left\{\begin{array}{lll}
j & j & i_{1} \\
j & I & i_{1}
\end{array}\right\}=\frac{1}{2}(5 j-3) /(4 j-1)(4 j-3) \\
& \left\{\begin{array}{lll}
j & j & i_{1} \\
j & I & i_{2}
\end{array}\right\}=-\frac{3}{2}\{(3 j-2)(j-1) /(4 j-5)(4 j-1)\}^{1 / 2} /(4 j-3) . \\
& \left\{\begin{array}{lll}
j & j & i_{2} \\
j & I & i_{2}
\end{array}\right\}=-\frac{1}{2} j /(4 j-3)(4 j-5)
\end{aligned}
$$

and thus

$$
\begin{aligned}
(1+X)_{\hat{\lambda}_{1} \lambda_{1}} & =3(3 j-2) /(4 j-3), \\
X_{\lambda_{1} \lambda_{2}}=X_{\hat{\lambda}_{2} \lambda_{1}} & =-3\{(3 j-2)(j-1)\}^{1 / 2} /(4 j-3) . \\
(1+X)_{\hat{\lambda}_{2} \lambda_{2}} & =3(j-1) /(4 j-3) .
\end{aligned}
$$

and

$$
\begin{aligned}
& M_{\lambda_{1} \lambda_{1}}=V_{\lambda_{1}}(5 j-3) /(4 j-3) . \\
& M_{\lambda_{1} \lambda_{2}}=-3 V_{\lambda_{2}}\{(3 j-2)(j-1)\}^{1 / 2} /(4 j-3) . \\
& M_{\lambda_{2} \lambda_{1}}=-3 V_{\lambda_{1}}\{(3 j-2)(j-1)\}^{1 / 2} /(4 j-3) . \\
& M_{\lambda_{2} \lambda_{2}}=-V_{\lambda_{2}} j /(4 j-3) .
\end{aligned}
$$

The pairon energies are

$$
\omega_{\lambda_{i}}=2 \varepsilon+V_{\lambda_{i}}
$$

where

$$
V_{\lambda_{i}}=2\left\langle j j \lambda_{i}|V| j j \hat{\lambda}_{i}\right\rangle .
$$

When solving the Dyson-like equation (4), we find zeros of det $\left(G_{0}^{-1}-M\right)$ at $\omega=3 \varepsilon$ and

$\omega=3 \varepsilon+\left\{(3 j-2) V_{\lambda_{1}}+(j-1) V_{\lambda_{2}}\right\} /(4 j-3)$.
The residue matrix of $\left(G_{0}^{-1}-M\right)^{-1}(1+X)$ is zero at the pole at $\omega=3 \varepsilon$. this being a spurious solution. At the second pole the residue matrix turns out to be equal to $1+X$. the trace of which is 3 (see eq. (10)). This normalization factor can be understood by realizing that in eq. (3) the labels $p_{1}$ and $p_{2}$ are not ordered relative to the constituents of $\pi_{1}$ and $\pi_{2}$. respectively. The dyadic decomposition of $(1+X) / 3$ gives vectors with two components which are the wellknown coefficients of fractional parentage (cfp) [5]. Thus for $I=3 j-3$. the spurious solution of $\operatorname{det}\left(G_{0}^{-1}-M\right)=0$ has vanishing residue and therefore does not appear in $G$. while the residue matrix of $G$ at the other solution supplies us with the cfp.

The above two cases are so simple that they need not be treated in terms of the formalism of references [1. 2]. Simple counting of $m$-states shows that no state with $I=3 j-4$ exists and only one state with $I=3 j-3$ can be formed and this has a simple prescribed configuration. We give these cases here because (i) they were discussed in reference [4] and. therefore, the difference in the two approaches is illustrated and (ii) this discussion shows that our approach embodies even cases for which it was not specifically designed. 
It was, in fact, designed for collective phenomena. Therefore, we now discuss the case of pairing. i.e. we consider the case $I=j$ and we set $V_{i}=0$ for $\lambda \neq 0$. The two-particle state with $\lambda=0$ has an energy $\omega_{0}=2 \varepsilon+V_{0}$. while those with $\lambda \neq 0$ have $\omega_{\lambda}=2 \varepsilon$. The mass operator $M$ has the structure

$$
\begin{aligned}
M_{\lambda \lambda^{\prime}} & =2 \hat{i}\left\{\begin{array}{lll}
j & j & \lambda \\
j & j & 0
\end{array}\right\} V_{0} \delta_{\lambda^{\prime}, 0} \\
& =\frac{2 \cdot \hat{\lambda}}{2 j+1} V_{0} \delta_{\lambda^{\prime}, 0}
\end{aligned}
$$

from which it follows that the three-particle solutions with $I=j$ are at $\omega=\varepsilon+\omega_{0}-2 V_{0} /(2 j+1)$ and $\omega=3 \varepsilon$, the latter being $\frac{1}{2}(2 j-1)$-fold degenerate. The former solution is shifted from its collective value $\varepsilon+\omega_{0}$ by a Pauli-correction term. At this pole the residue $R_{\lambda \lambda^{\prime}}$ turns out to be

$$
\begin{aligned}
& R_{00}=1+X_{00} . \\
& R_{0 \lambda}=R_{\lambda 0}=X_{0 \lambda} . \quad \forall \hat{\lambda} \neq 0 \\
& R_{\lambda \lambda^{\prime}}=\frac{X_{0 \lambda} X_{0 \lambda^{\prime}}}{1+X_{00}} . \quad \forall \lambda . \lambda^{\prime} \neq 0 \\
& X_{0 \lambda}=-2 \hat{i} /(2 j+1) .
\end{aligned}
$$

For $j=\frac{1}{2}$ the residue is zero. otherwise the trace of the residue matrix $\operatorname{tr}(R)$ is again 3 . the reason being the same as in the case with $I=3 j-3$ discussed above. The matrix $\frac{1}{3} R$ is decomposed into a dyadic form and a vector $r$ with elements

$$
\begin{aligned}
& r_{0}=\{(2 j-1) /[3(2 j+1)]\}^{1 / 2} . \\
& r_{\lambda}=-2 \hat{\lambda} /\{3(2 j-1)(2 j+1)\}^{1 / 2} . \quad \forall \lambda \neq 0
\end{aligned}
$$

is obtained. These elements of $r$ are the cfp for the seniority-one state.

The residue matrix $\bar{R}$ for the pole at $\omega=3 \varepsilon$ is

$$
\begin{aligned}
& \bar{R}_{00}=\bar{R}_{0 \hat{\lambda}}=\bar{R}_{\lambda 0}=0 \text {. } \\
& \bar{R}_{\hat{\lambda} \lambda^{\prime}}=\delta_{\lambda \lambda^{\prime}}+X_{\lambda \lambda^{\prime}}-\frac{X_{\lambda 0} X_{0 \hat{\lambda}^{\prime}}}{1+X_{00}} . \quad \forall \hat{\imath} . \hat{\iota}^{\prime} \neq 0
\end{aligned}
$$

and this is zero for $j \leqslant \frac{7}{2}$. while it has non-zero elements for $j \geqslant \frac{9}{2}$. The trace of the residue matrix $\operatorname{tr}(\bar{R})$ has the value 3 for $j=\frac{9}{2}, \frac{11}{2}$ and $\frac{13}{2}$. and the value 6 for the next three half-integer values. etc. When $\operatorname{tr}(\bar{R})=3$, there is only one state of seniority $v=3$ and a dyadic decomposition of $\frac{1}{3} \bar{R}$ yields a vector the components of which are the cfp of this state. For $j \geqslant \frac{15}{2}$, two or more states with $v=3$ are found and an additional quantum number is necessary to specify the states [6].

As in reference [1] the above method can be extended to the propagation of two pairons. This leads to expressions similar to those obtained in reference [7] by the Dyson Boson expansion method [8]. The results of reference [7] show that drastic truncation is possible in realistic calculations.

In summary it is pointed out that the overcompleteness of the basis causes no problems; the unphysical solutions all have zero residues while the dimension of the residue matrices coincide with the dimension of the vectors representing the coefficients of fractional parentage. That this is so in general. can be seen by studying the spectral representation of $G$ defined in eq. (3).

The authors wish to thank Dr. C. A. Engelbrecht for valuable discussions.

\section{References}

[1] Hahne, F. J. W., Phys. Lett. 73B (1978) 239.

[2] Hahne, F. J. W., Phys. Lett. 74B (1978) 149.

[3] Bortignon, P. F., Broglia, R. A., Bes, D. R. and Liotta, R., Phys. Rept. 30 (1977) 305.

[4] Liotta, R. J. and Silvestre-Brac, B., J. Physique Lett. 39 (1978) L-11.
[5] De Shalit, A. and Talmi, I., Nuclear Shell Theory (Academic Press, New York) 1963, p. 268.

[6] Bayman, B. F. and Lande. A., Nucl. Phys. 77 (1966) 1.

[7] Schuck, P., Wittmann, R. and Ring, P., Lett. Nuovo Cimento 17 (1976) 107.

[8] Ring, P. and Schuck, P., Phys. Rev. C 16 (1977) 801. 